\title{
Research on Support Vector Machines Method Modeling for Rice Potassium Nutrition Diagnosis
}

\author{
Qiong Zhou ${ }^{1,3}$, Hongyun Yang ${ }^{2,3, *}$, Jun Yang ${ }^{2}$, Yuting Sun ${ }^{1,3}$, Aizhen Sun ${ }^{3}$ and Wenji Yang ${ }^{2,3}$ \\ ${ }^{1}$ School of Computer and Information Engineering, Jiangxi agricultural university, Nanchang, Jiangxi, China \\ ${ }^{2}$ College of Software Engineering, Jiangxi agricultural university, Nanchang, Jiangxi, China \\ ${ }^{3}$ Key Laboratory of Agricultural Information Technology of Colleges and Universities in Jiangxi Province, Nanchang, Jiangxi, \\ China \\ ${ }^{*}$ Corresponding author
}

\begin{abstract}
A machine learning method was used to establish a diagnostic model of potassium nutrition for rice obtained from image processing techniques. In this study, super hybrid rice "Liangyoupeijiu" was used as experimental object to set up four kinds of rice cultivation experiments at different potassium fertilization levels, the image data of a total of 1920 groups of the 1st leaves and the 2nd leaves, the 3rd leaves, and their corresponding sheaths were obtained by scanning with a scanner. Nineteen rice characteristic indexes were obtained. Support vector machine was used to establish the diagnostic model of potassium nutrition in nineteen rice characteristic indexes, and to diagnose and identify the potassium nutrition in rice. The experimental results show that the identification method based on image processing and SVM is suitable for the diagnosis of potassium nutrition of the 3rd leaves in rice young panicle differentiation stage with an accuracy of $89 \%$, which provides a reliable and universal method for studying the recognition of potassium nutrition in rice and can meet the needs of agronomic research.
\end{abstract}

Keywords-rice; potassium nutrition diagnosis; image processing; support vector machines

\section{INTRODUCTION}

As the main food crops in China, rice has been steadily invested in chemical fertilizers to achieve its high yield and stable yield. The rational application of chemical fertilizers has become a prerequisite for the high and stable yield of rice[1]. The results of the pilot farm survey conducted by Fuyang Scientific Research Base of China National Rice Research Institute also showed that about $90 \%$ of rice has different symptoms of potassium deficiency[2]. Therefore, the application of potassium fertilizer has become one of the key measures for rice yield[3]. Using machine learning method to establish the diagnostic model of potassium nutrition for rice from image processing techniques, which is different from the traditional empirical statistical model modeling method to quickly and accurately judge the nutritional status of crop growth and the inversion of crop growth process by computer vision technology, and to lay the foundation for the crop potassium management at the same time provide a scientific theoretical basis. In recent years, image processing technology has been widely used in agriculture. Li Ming[4] used the improved dynamic threshold segmentation method in image processing to extract the leaf area of wheat in the image and calculate the leaf area index, the absolute difference with the true area value was less than 0.1 , and the effect was good; Wang Shuwen[5] used image processing to obtain the diseased area of cucumber leaf and used BP neural network to classify the diseases. The average recognition accuracy reached $95.31 \%$. Zhang Nannan[6] used the image processing to detect the mildewed corn particles and the correct detection rate of more than 87.5\%; Wu Lulu[7] extracted rice blast lesion by image processing, the recognition rate of $90.26 \%$, the effect was better In recent years, former scholars began to study the status of rice potassium nutrition diagnosis[8-10]. Shi Yuanyuan[8-9] used the image processing to obtain the color, shape and texture characteristics of rice leaves for the quantitative diagnosis of leaf potassium. It was found that the third fully expanded leaf was better than the first fully expanded leaf and the accuracy was evaluated by the error analysis matrix method. Its overall recognition accuracy was $96 \%$, and the effect was better. Chen Lisu[10] carried out potted rice experiment, obtained the characters of spectral, space and shape of rice leaves by image processing and established model of diagnosis of rice potassium nutrition.

The application of support vector machine in multiple growth stages of the diagnosis of rice potassium nutrition has not yet appeared. In this study, rice experiments with different levels of potash (equivalent to $0 \mathrm{~kg} \cdot \mathrm{hm}^{-2}, 210 \mathrm{~kg} \cdot \mathrm{hm}^{-2}, 300$ $\mathrm{kg} \cdot \mathrm{hm}^{-2}, 390 \mathrm{~kg} \cdot \mathrm{hm}^{-2}$ ) $\mathrm{Y}_{1}, \mathrm{Y}_{2}, \mathrm{Y}_{3}$ and $\mathrm{Y}_{4}$ were set up. The images of the 1st leaves, the 2nd leaves, the 3rd leaves and corresponding sheaths of rice at different stages were obtained by scanner scanning. Nineteen rice characteristics were obtained by using image processing technology. Nineteen rice characteristic data were used to establish the rice potassium nutrition diagnostic model for the diagnosis of rice potassium nutrition identification. To explore the diagnostic accuracy of potassium nutrition in different periods of rice and to find out the feasible method for accurately diagnosing the nutrition of rice. It provides a new way to study the accurate and rapid identification of potassium in rice.

\section{Materials AND MethodS}

\section{A. The Original Data of the Experiment}

\section{1) The Experiment Site}

The experiment site was located at Chengxin Farm (116.15'E, 28.92'N) in Nanchang, Jiangxi Province, China. The physical and chemical properties of soil were as follows: $\mathrm{pH}$ 
5.3, total potassium $1.4 \mathrm{~g} / \mathrm{kg}$, organic matter $24.4 \mathrm{~g} / \mathrm{kg}, 12.7$ $\mathrm{mg} / \mathrm{kg}$, available potassium $123.0 \mathrm{mg} / \mathrm{kg}[11]$.

\section{2) The Experiment Design}

With super hybrid rice - "Liangyoupeijiu" as the test material, sown on May 25, 2017, the same year on June 14 transplanted. As the experiment is high-yielding rice cultivation experiment, reference[12] mentioned that when the potash application rate was $270 \mathrm{~kg} / \mathrm{hm}^{2}$, the yield increase was better than that of $135 \mathrm{~kg} / \mathrm{hm}^{2}$. Therefore the four potassium levels were set at $0 \mathrm{~kg} \cdot \mathrm{hm}^{-2}, 210 \mathrm{~kg} \cdot \mathrm{hm}^{-2}, 300 \mathrm{~kg} \cdot \mathrm{hm}^{-2}, 390 \mathrm{~kg} \cdot \mathrm{hm}^{-2}$. The potassium applied was converted into urea and applied according to the ratio of basal fertilizer: tillering fertilizer: panicle fertilizer $=2: 1: 2$. One day before transplanting rice applied basal fertilizer, transplanting applied tillering on the 7th day, young panicle differentiation stage IV application of panicle fertilizer. Repeated three times. Other management measures were in accordance with the daily management of rice cultivation methods.

\section{3) Acquire the Rice Image}

The rice images used in this study were screened by MICROTEK scanner (model MRS-9600TFU2L with a resolution of $600 \mathrm{ppi}$ ) during panicle differentiation stage (July 24, 2017) and full heading stage (August 23, 2017), a total of $19202515 * 3997$ pixel rice images. Among them, the images of the 1st leaves, the 2nd leaves and the 3rd leaves of rice in each period were 320 (the corresponding leaf sheath image was included in the leaf image).

\section{B. Rice Characteristic Data}

\section{1) Feature Selection}

In this study, nineteen characteristics, including leaf color and leaf shape, were selected as the input characteristics of rice potassium nutrition diagnosis model. They were rice leaf $R$, leaf $G$, leaf $B$, leaf $H$, leaf $S$, leaf $I$, leaf sheath $R$, leaf sheath $G$ Leaf sheath B, Leaf sheath $H$, Leaf sheath $S$, Leaf sheath I, Leaf length, Maximum leaf width, area, circumference, area perimeter ratio, area length ratio, eccentricity (length to maximum width ratio).

2) Rice Image Processing

The image processing program is implemented using MATLAB R2014a. The RGB color images obtained by the scanner are converted to HSV images. By comparing the six channel images of R, G, B, H, S and V, as shown in figure 1, it was found that rice leaves under $\mathrm{H}$ channel were the most distinguishable from the background.
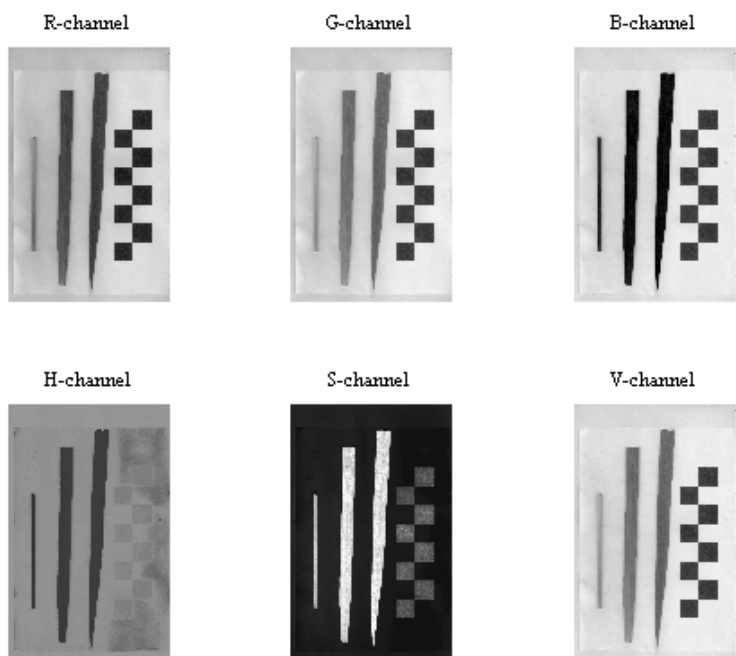

\section{FIGURE I. THE CONTRASTIVE CHART OF CHANNEL IMAGE}

$\mathrm{H}$-channel images were transformed into binary images using threshold method, in which the threshold value was set to 0.3 , and the images were color inverted for the convenience of subsequent processing. Then imfill function and bwareaopen function were used to remove the small area in rice leaves and background Interference area, as shown in Figure 2.

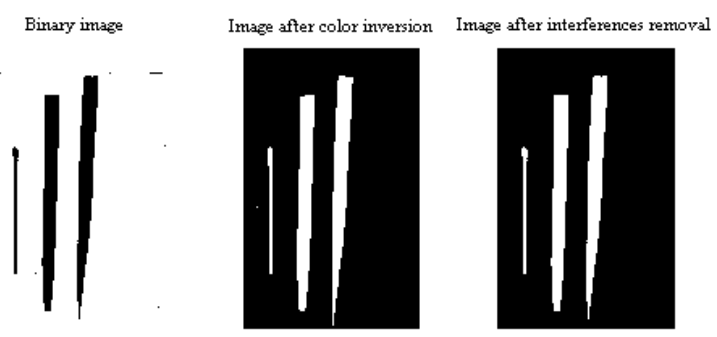

FIGURE II. THE CHART OF REMOVING IMPURITIES

For rice leaf color features, the image of the rice with a black background was obtained by dot-multiplying the image with the original image by the cat function. The bwlabel function was used to mark the connected domain. The regionprops function was used to determine the corresponding positions of leaf sheaths and leaves. The rice image was divided into rice leaf images and rice leaf sheath images. As shown in Figure 3, the images were converted into HSI color space images. rice leaf images and rice leaf sheath images were averaged for R, G, B, H, S, I. 


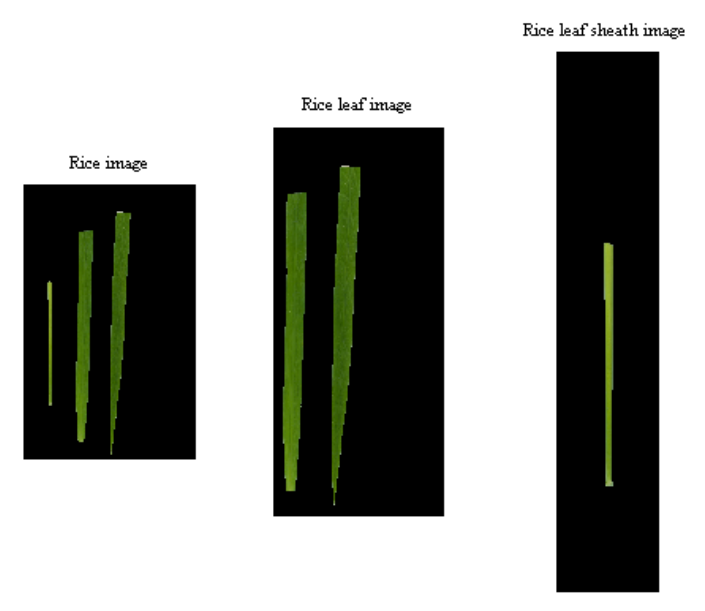

FIGURE III. THE BLACK BACKGROUND CHART

For the morphological characteristics of rice leaves, eight square reference objects (the actual side length of $2.5 \mathrm{~cm}$ ) were used as calculation references for morphological parameters of rice leaves and the minimum circumscribed rectangles of rice leaf images and reference images were respectively defined as shown in Figure 4. Among them, the length, width, circumference and area of rice leaves were calculated according to (1), (2), (3) and (4) respectively.
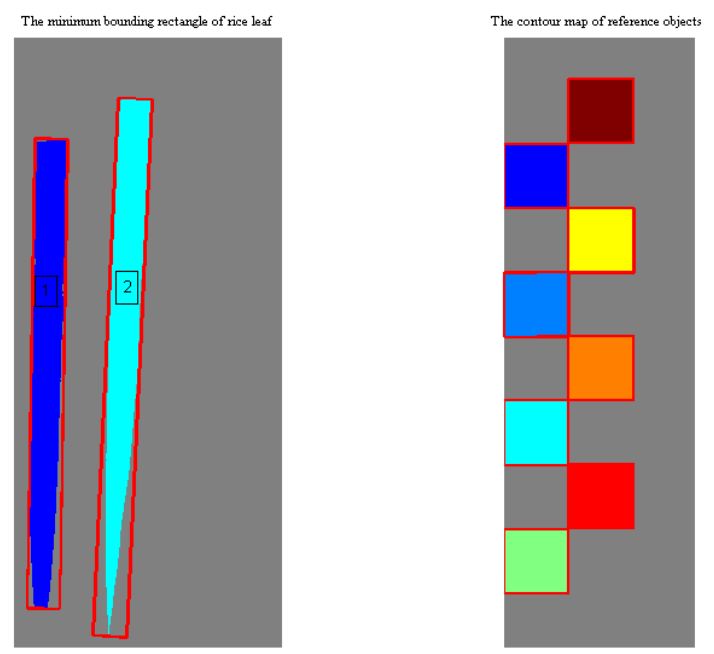

FIGURE IV. THE CHART OF THE SMALLEST EXTERNAL RECTANGLE

$$
\text { Len }=\frac{\left(\operatorname{Len}_{1}+\operatorname{Len}_{2}\right) * \text { Len }_{\text {actual }}}{\operatorname{Len}_{\text {refer }}}
$$

As is shown in (1), Len represents rice leaf length, Len $_{1}$ and $\mathrm{Len}_{2}$ are the number of pixels of the minimum circumscribed rectangle length of the leaf 1 and the leaf 2 , $L e n_{\text {refer }}$ is the number of pixels of the total length of the 8 square reference objects, and $L^{2 e n_{\text {actual }}}$ is the total length of the 8 square reference objects.

$$
\text { Wid }=\frac{M A X\left(W i d_{1}, W i d_{2}\right) * \operatorname{Len}_{\text {actual }}}{\operatorname{Len}_{\text {refer }}}
$$

As is shown in (2), Wid is the max width of rice leaf, MAX() represents the maximum value, $W_{i d}$ is the number of pixels of the minimum circumscribed rectangle width of the leaf 1, Wid $_{2}$ is the number of pixels of the minimum circumscribed rectangle width of the leaf 2 .

$$
\text { Cir }=\frac{\left(\operatorname{Cir}_{1}+\operatorname{Cir}_{2}-W_{i d_{1}}-W_{i d}\right) * \operatorname{Len}_{\text {actual }}}{\operatorname{Len}_{\text {refer }}}
$$

As is shown in (3), Cir is the rice leaf circumference, $\mathrm{Cir}_{1}$ represents the total number of pixels of the leaf 1 's edge, and $\mathrm{Cir}_{2}$ represents the total number of pixels of the leaf 2's edge.

$$
\text { Area }=\frac{\left(\text { Area }_{1}+\text { Area }_{2}\right) * \text { Area }_{\text {actual }}}{\text { Area }_{\text {refer }}}
$$

As is shown in (4), Area is the rice leaf area, Area ${ }_{1}$ and Area $_{2}$ are the number of pixels of leaf 1 and leaf 2 . Area actual represents the actual total area of 8 square reference objects, and Area $_{\text {refer }}$ represents the number of pixels of total area of 8 square reference objects.

\section{Establishment of a Diagnostic Model of Rice Potassium Nutrition}

In the present study, a diagnostic model of the rice potassium nutrition was established to diagnose and identify the rice potassium nutrition by using support vector machine. Nineteen rice characteristic data were used as input parameters of the model. Four potassium nutrition levels, $\mathrm{Y}_{1}, \mathrm{Y}_{2}, \mathrm{Y}_{3}$ and $\mathrm{Y}_{4}$, were used as output parameters of the model. Output parameters were classified into four categories according to different levels of potassium nutrition: $[0,0,0,0][0,1,0,0]$ $[0,0,1,0] \quad[0,0,0,1]$ respectively represented 4 levels of potassium nutrition $\mathrm{Y}_{1}, \mathrm{Y}_{2}, \mathrm{Y}_{3}$ and $\mathrm{Y}_{4}$. The diagnostic tests of potassium nutrition at different leaf positions (the 1st leaves, the 2nd leaves and the 3rd leaves) at different stages (young panicle differentiation stage and full heading stage) were compared.

\section{1) Data Normalization}

In order to eliminate the dimensionality effect between rice characteristic index data, the data were standardized to solve the comparability of data indexes. This test uses the mapminmax function to normalize the test data. Mapminmax function used by the mapping as shown in (5):

$$
y=\frac{\left(y_{\max }-y_{\min }\right) \cdot\left(x-x_{\min }\right)}{\left(x_{\max }-x_{\min }\right)}+y_{\min }
$$

$X \min$ and $X \max$ are the minimum and maximum values of the feature data set $X, Y \min$ and Ymax are the mapped range parameters. In this experiment $Y \min =-1, Y \max =1$. 


\section{2) Support Vector Machines}

Support vector machines(SVM) has its unique advantages in solving nonlinear small sample data sets, high-dimensional spatial pattern recognition problem[13-14]. Therefore, it was widely used by scholars in the field of crop[15-18]. The idea of SVM is to construct a soft separation hyperplane in the feature space determined by the kernel function so that the classification surface with the largest classification interval is the optimal classification surface. Using the quadratic programming method, the optimal decision function can be obtained as shown in (6), is the Lagrange multiplier, is the category label, is the category label and is the kernel function[19].

$$
f(x)=\operatorname{sgn}\left(\sum_{i=1}^{m} \alpha_{i}^{*} y_{i} k\left(x_{i}, x\right)+b\right)
$$

In the SVM model, the kernel function was the theoretical basis of the SVM method. There were four commonly used kernel functions: linear kernel function, polynomial kernel function, radial basis function and signoid kernel function[19]. The overall classification accuracy of SVM model under different kernel functions for the 3rd leaves fertilization at different periods was shown in Table 1 . It could be found that for the 3rd leaves data of young panicle differentiation stage and full heading stage, the diagnostic accuracy of rice potassium nutrition diagnosis was the highest with SVM model
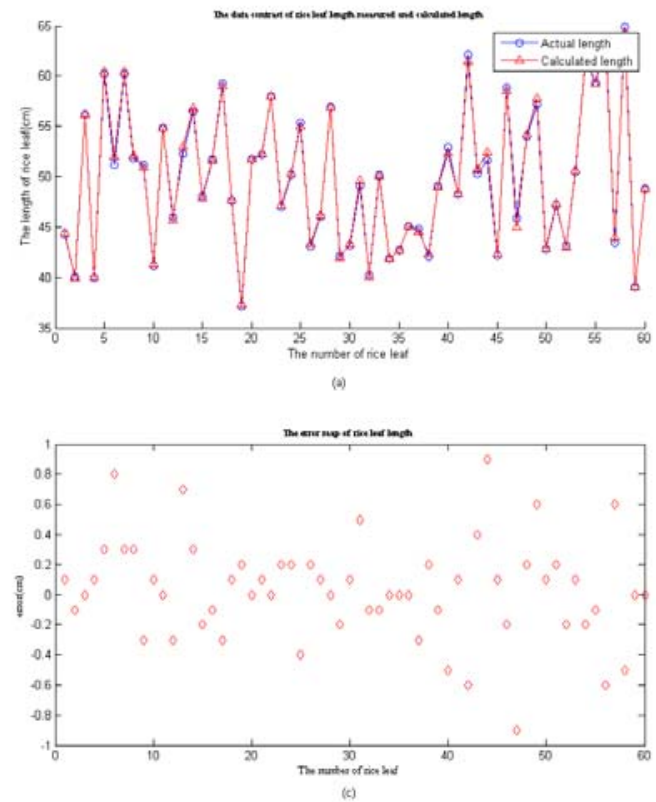

FIGURE V. THE CHART OF LENGTH AND WIDTH ERROR COMPARISON

\section{B. Analysis of Diagnosis and Identification of Potassium} Nutrition in Rice

The support vector machine was used to establish a diagnostic model of rice potassium nutrition based on the nineteen rice characteristics obtained above. The results showed in Table 2. For the diagnosis model of potassium based on radial basis function. Therefore, radial basis function was chosen as the kernel function of SVM model in this study.

TABLE I. THE CLASSIFICATION ACCURACY OF POTASSIUM NUTRITION LEVEL BY SVM MODEL UNDER DIFFERENT KERNEL FUNCTIONS

\begin{tabular}{|l|l|c|}
\hline \multicolumn{1}{|c|}{ Kernel Function } & $\begin{array}{c}\text { Young Panicle } \\
\text { Differentiation Stage }\end{array}$ & $\begin{array}{c}\text { Full Heading } \\
\text { Stage }\end{array}$ \\
\hline Linear kernel function & $88 \%(88 / 100)$ & $67 \%(67 / 100)$ \\
\hline polynomial kernel function & $88 \%(88 / 100)$ & $58 \%(58 / 100)$ \\
\hline radial basis function & $89 \%(89 / 100)$ & $68 \%(68 / 100)$ \\
\hline signoid kernel function & $39 \%(39 / 100)$ & $44 \%(44 / 100)$ \\
\hline
\end{tabular}

\section{RESULTS AND ANALYSIS}

\section{A. Error Analysis of Rice Leaf Length and Width}

In order to judge and analyze the accuracy of morphological characteristics of rice leaf length and maximum width in this experiment, 60 leaves were randomly selected during the scanning process for manual measurement. The length and width of the image processing and corresponding artificial length and width measurement results and the corresponding error graph was shown in Fig.6. The length of rice leaves ranged from $37.1 \mathrm{~cm}$ to $64.9 \mathrm{~cm}$ and the maximum width ranged from $1.4 \mathrm{~cm}$ to $2.2 \mathrm{~cm}$. The error of rice length mainly concentrated in $0 \mathrm{~cm} \sim 0.3 \mathrm{~cm}$, the maximum error was $0.9 \mathrm{~cm}$, the minimum error was $0 \mathrm{~cm}$, The maximum width of the error is mainly concentrated in $0 \mathrm{~cm} \sim 0.1 \mathrm{~cm}$, the maximum error of $0.2 \mathrm{~cm}$, the minimum error of $0 \mathrm{~cm}$, the error is small, the effect is better, to meet the study needs.
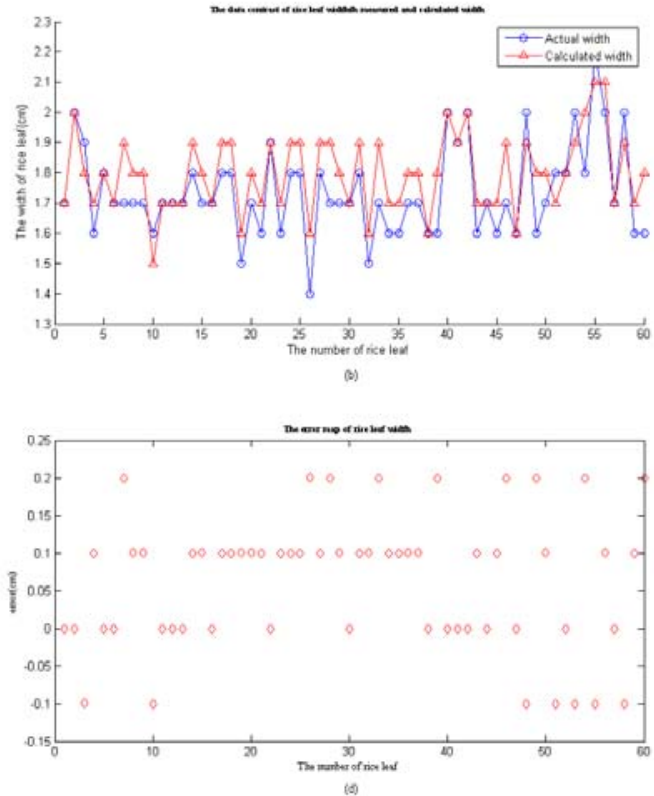

nutrition established by support vector machines, the overall recognition accuracy of rice in young panicle differentiation stage and full heading stage was the 1st leaves, the 2nd leaves, the 3rd leaves. Compared with full heading stage, the overall recognition accuracy of the 1st leaves to the 3rd leaves in young panicle differentiation stage were $20 \%$, 20\% and $21 \%$ 
higher than that of the control. The overall recognition accuracy of the 3rd leaves was the highest in young panicle differentiation stage, and the accuracy rate of support vector machine model was $89 \%$.

\section{TABLE II. THE OVERALL RECOGNITION ACCURACY RATE OF} POTASSIUM NUTRITION DIAGNOSIS

\begin{tabular}{|c|c|c|c|c|c|c|}
\hline \multirow{2}{*}{$\begin{array}{c}\text { Modeli } \\
\text { ng } \\
\text { Metho } \\
\text { d }\end{array}$} & \multicolumn{3}{|c|}{$\begin{array}{c}\text { Young Panicle } \\
\text { Differentiation Stage }\end{array}$} & \multicolumn{3}{c|}{ Full Heading Stage } \\
\cline { 2 - 7 } & $\begin{array}{c}\text { The 1st } \\
\text { leaves }\end{array}$ & $\begin{array}{c}\text { The 2nd } \\
\text { leaves }\end{array}$ & $\begin{array}{c}\text { The 3rd } \\
\text { leaves }\end{array}$ & $\begin{array}{c}\text { The 1st } \\
\text { leaves }\end{array}$ & $\begin{array}{c}\text { The } \\
\text { 2nd } \\
\text { leaves }\end{array}$ & $\begin{array}{c}\text { The } \\
\text { 3rd } \\
\text { leaves }\end{array}$ \\
\hline SVM & $84 \%$ & $85 \%$ & $89 \%$ & $64 \%$ & $65 \%$ & $68 \%$ \\
\hline
\end{tabular}

The application of support vector machine to establish a model of diagnosis of potassium in the diagnosis of potassium, the third and fourth category of the highest recognition rate of $96 \%$, followed by the first category, 88\%,The second type had the poorest identification effect, which was $76 \%$. The four potassium nutrition levels were replaced by 1,2,3,4 of the test category label in turn, the ordinate was the label of the level of potassium nutrition level, and the sequence number of the abscissa test sample, in which the actual classification and prediction classification map of the top trefoil in young panicle differentiation stage is shown in Figure 7, it can be found that the second category is easily mistaken for the third category, the first category has three categories mistaken for the second category, the third category and the fourth category have only one classification error.

\section{TABLE III. THE IDENTIFICATION ACCURACY RATE OF POTASSIUM} NUTRITION DIAGNOSIS

\begin{tabular}{|l|c|c|c|c|}
\hline \multirow{2}{*}{$\begin{array}{c}\text { Modeling } \\
\text { Method }\end{array}$} & \multicolumn{4}{|c|}{ The 3rd Leaves In Young Panicle Differentiation Stage } \\
\cline { 2 - 5 } & $\begin{array}{c}\text { The first } \\
\text { category }\end{array}$ & $\begin{array}{c}\text { The second } \\
\text { category }\end{array}$ & $\begin{array}{c}\text { The third } \\
\text { category }\end{array}$ & $\begin{array}{c}\text { The fourth } \\
\text { category }\end{array}$ \\
\hline SVM & $88 \%$ & $76 \%$ & $96 \%$ & $96 \%$ \\
\hline
\end{tabular}

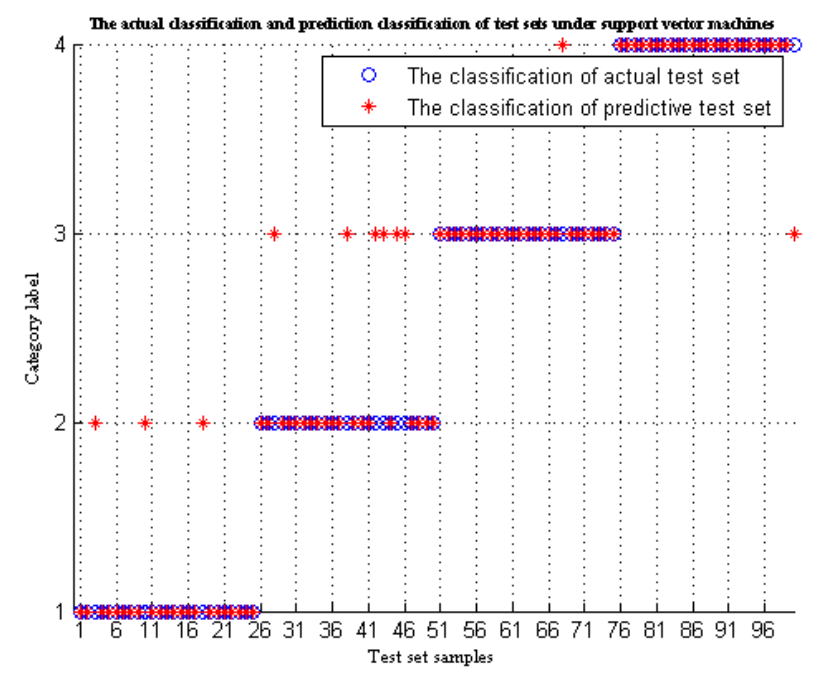

FIGURE VI. THE RECOGNITION ACCURACY RATE BASED ON SVM OF DIAGNOSIS NUTRITION DIAGNOSIS

\section{CONCLUSION}

In this study, the image processing method was used to obtain the characteristic data of rice. The error of calculating the length of rice leaf mainly concentrated in $0 \mathrm{~cm} \sim 0.3 \mathrm{~cm}$. The error of the maximum width of rice leaf mainly concentrated in $0 \mathrm{~cm} \sim 0.1 \mathrm{~cm}$ with small error, strong feasibility.

In this study, the color and morphological characteristics of 19 rice cultivars were obtained by image processing. By using support vector machine (SVM) in machine learning, the characteristics of rice were identified and diagnosed by potassium nutrition analysis. The results showed that the differentiation characteristics of the 3rd leaves, Which is easy to diagnose and recognize potassium nutrition. The overall recognition accuracy rate is $89 \%$. The first category is completely recognized correctly, the third category and fourth category are misidentified once, and the second category is easily confused with the third category.

In this study, the input characteristics of rice potassium nutrition diagnostic model were obtained by the image technology. Different from the traditional modeling methods, the machine learning method was used to establish the diagnosis model of potassium nutrition in rice, which laid a theoretical foundation for the rapid and accurate acquisition of the crop nutritional status by computer vision, provide support for the virtual research of crop growth inversion and provide an effective and feasible method for crop potassium nutrition diagnosis and identification, which is of great significance for scientific guidance on crop fertilization.

\section{ACKNOWLEDGEMENTS}

National Natural Science Foundation Project: (No.61562039), (No.61363041), (No.61462038)

Jiangxi Provincial Department of Education Science and Technology Project:(No.GJJ160374), (No.GJJ170279)

\section{REFERENCES}

[1] Qiangsheng Wang, Accumulation characteristics and physiological effect of rice potassium nutrition. Nanjing: Nanjing Agricultural University, 2009.

[2] Ruiqing Zhou, "Effects of fertilization on rice quality and yield," Changsha: Journal of Hunan Agricultural University(Natural Sciences), vol. 15, pp. 1-5, September 1989.

[3] Yuchuan Ding, Wei Luo, Guohua $\mathrm{Xu}$, "Effects of $\mathrm{Mg}, \mathrm{K}$ and their interaction on grain yield and yield components and nutriment uptake of rice,” Xianyang: Journal of soil and water conservation, vol. 22, pp. 178182, June 2008.

[4] Ming Li, Changli Zhang, Junlong Fang, "Extraction of leaf area index of wheat based on image processing," Beijing: Transactions of the CSAE, vol. 26, pp. 205-209.

[5] Shuwen Wang, Changli Zhang "Study on identification of cucumber leaf disease based on image processin," Harbin: Journal of northeast agricultural university, vol. 43, pp. 69-73, May 2012.

[6] Nannan Zhang, Wei Liu, Wei Wang, Xinzhi Ni, Xuan Zhu, "Image processing method of corn kernels mildew infection and aflatoxin levels," Beijing: Journal of the chinese cereals and oils association, vol. 29, pp. 82-88, February 2014.

[7] Lulu Wu, Zhixiong Zheng, Long Qi, Xu Ma, Zhongwei Liang, Guorui Chen, "Detection method of rice leaf blast based on image processing," Harbin: Journal of agricultural mechanization research, vol. 9, pp. 32-35, September 2014.

[8] Yuanyuan Shi, Jisong Deng, Dongyan Zhang, Xiaodong Ding, Ke Wang, "Leaf characteristics extraction of rice under potassium stress based on static scan and spectral segmentation technique," Beijing: Spectroscopy and spectral analysis, vol. 30, pp. 214-219, January 2010. 
[9] Yuanyuan Shi, Rice nutrition diagnosis and modeling based on digital image. Hangzhou: Zhejiang University, 2010.

[10] Lisu Chen, Rice nutrition identification and diagnosis based on machine vision technology. Hangzhou: Zhejiang University, 2014.

[11] Yangyang Zhang, Jianwei Lu, Youzhu Wang, Zhen Wang, Xiaokun Li, Tao Ren, Rihuan Cong, "Effects of potassium fertilizer application method on yield and potassium apparent efficiency of direct-sowing rice and trasplanting rice,” Beijing: Crops, vol. 1, pp. 110-114, January 2016.

[12] Huihuang Cheng, Qinyin Shang, Zhenbo Yi, Houliang Zheng, Yongjun Zeng, "Effects of fertilizer application rate on yield and population quality of super hybrid rice at different yield levels,” Hangzhou: China rice, vol. 23, pp. 81-88, April 2017.

[13] C. Cortes, V.N.Vapnik, "Support vector networks," Mach. Learn-ing, vol. 20, pp. 273-297, 1995.

[14] S. Liu, N. Jiang, "SVM parameters optimization algorithm and its application,” IEEE International Conference on Mechatronics and Automation, vol.14, pp. 509-513, March 2009.

[15] R. Kaundal, A. S. Kapoor, G. P. Raghava, "Machine learning techniques in disease forecasting: A case study on rice blast prediction,” London: Bmc Bioinformatics, vol. 7, pp. 1-16, July 2006.

[16] Jie Lv, Fengli Deng, Zhenguo Yan, "Using PROSEPCT and SVM forthe Estimation of Chlorophyll Concentration," Advanced Materials Research, vol. 989-994, pp. 2184-2187, July 2014.

[17] Kaicai Zhao, Research on technology of rice blast recognition based on support vector machines. Harbin:Harbin Engineering University, 2012.

[18] Jingjing Shi, Zhanyu Liu, Lili Zhang, Wan Zhou, Jingfeng Huang, "Hyperspectral recognition of rice damaged by rice leaf roller based on Support Vector Machine,” Beijing: Chinese Journal Of Rice Science, vol. 23, pp. 331-334, March 2009.

[19] Rongjie Song, Jifeng Ning ,Xiuying Liu, Qinrui Chang, “Apple orchard extraction with quickbird imagery based on texture features and support vector machine," Beijing: Transactions of the Chinese Society for Agricultural Machinery, vol. 48, pp. 188-197, March 2017.

[20] Hongyun Yang, Aizhen Sun, Huojiao He, "Study on the geometry parameter of rice leaf measuring method using image vision technology,” Wuhan: Hubei Agricultural Sciences, vol. 54, pp. 43174320, September 2015.

[21] Fusong Huang, Zongxiu Sun, Peisong Hu, Shaoqing Tang, "Present situations and prospects for the research on rice grain quality forming," Beijing: Chinese journal of rice science, vol. 12, pp. 172-176, December 1998.

[22] Weini Wang, Jianwei Lu, Yuqing He, Xiaokun Li, Hui Li, "Effects of N, $\mathrm{P}, \mathrm{K}$ fertilizer application on grain yield, quality, nutrient uptake and utilization of rice," Beijing: Chinese journal of rice science, vol. 25, pp. 645-653, November 2011.

[23] Shiwei Guo, Xueqiang Zhao, Shijian Xia, Hongxia Zh, Weiming Shi, "The leaf and root nourishment physiology research for the super-hybrid rice after heading,” Beijing: Acta Agriculturae Boreali-Sinica, vol. 29, pp. 115-121, March 2014. 